\section{Review: use of pedometers increases physical activity in adults}

\section{QUESTION}

In adult outpatients, does the use of pedometers increase physical activity?

\section{REVIEW SCOPE}

Selected studies assessed pedometer use among $>5$ adult outpatients. Studies were excluded if participants were admitted to hospital or confined to a research centre, pedometers were sealed so that participants in the intervention group could not see the number of steps, or pedometers were used to measure the effects of a drug on a person's ability to be physically active.

Outcome was change in number of steps walked daily.

\section{REVIEW METHODS}

Medline (to Feb 2007), EMBASE/Excerpta Medica, Sport Discus, PsycINFO, Cochrane Library, Thompson Scientific, and ERIC (to May 2006); conference proceedings; and reference lists were searched for English-language studies. Experts in exercise physiology were consulted. 8 randomised controlled trials (RCTs) $(\mathrm{n}=305)$ and 18 observational studies $(n=2462)$ were included (mean age $49 y, 85 \%$ women). Studies were done in the USA or Canada (20 studies), Japan (2 studies), Europe (2 studies), or Australia (2 studies). Duration of the physical activity intervention ranged from 3 to 104 weeks (mean $18 \mathrm{wk}$ ).

\section{MAIN RESULTS}

In 8 RCTs, use of pedometers increased the number of steps walked daily; statistical heterogeneity was present, but the effect persisted even after removal of 1 study that showed a much higher increase in physical activity (table).

\section{CONCLUSION}

In adults, use of pedometers increases physical activity.

A modified version of this abstract appears in Evidence-Based Nursing

\section{ABSTRACTED FROM}

Bravata DM, Smith-Spangler C, Sundaram V, et al. Using pedometers to increase physical activity and improve health: a systematic review. JAMA 2007;298:2296-304.

Correspondence to: Dr D M Bravata, Primary Care and Outcomes Research, Stanford, CA, USA; dbravata@stanford.edu

Source of funding: National Institute on Aging and National Science Foundation.

- Clinical impact ratings: GP/FP/Primary care 6/7; Endocrine 5/7

Use of pedometers plus advice to record daily steps $v$ no pedometer or obscured pedometer in adults*

ncreasing physical activity in sedentary adults is difficult to achieve and harder to sustain. We need more effective strategies in primary health care. Pedometers cost little and provide objective feedback to users, avoiding the tendency to overestimate usual activity. It is encouraging to see that the use of pedometers may increase physical activity, albeit in the short term.

The follow-up time of the 8 RCTs in this metaanalysis ranged from 6 to 24 weeks, with most studies assessing participants at $\leqslant 12$ weeks, so there is no information yet about the sustainability of changes in activity levels. None of the RCTs assessed the effect of pedometers alone. The interventions included additional step goals, activity diaries, 1-4 physical activity counselling sessions, or monthly phone reminders. Giving a patient a pedometer alone may not change behaviour without providing step/ activity goals, diaries, or additional counselling and support.

It is hard to say whether a 2000-step increase would translate to health gains, although there is some evidence it may. ${ }^{1}$ Furthermore, what step goal should physicians advise? The 10000 step/day goal is popular and equates to about $300-400 \mathrm{kcal} /$ day energy expenditure, while the recommended goal of 30 minutes of walking/day (3000-4000 steps) equates to about $150 \mathrm{kcal} /$ day. However, this recommendation entails 30 minutes of walking over and above usual daily activities, whereas the daily step count includes all incidental walking, so the health gains may be similar. ${ }^{1}$ But 10000 steps may not be suitable for everyone, particularly people who were previously sedentary, older people, or those with physical limitations, and it may be off-putting. The daily number of steps of "healthy" adults ranges from 7000 to 13000 and may even be less in older people. ${ }^{1}$

Even so, pedometers with goal setting or counselling may be better than goal setting or counselling alone, although this needs further research to confirm. RCTs with longer follow-up of $\geqslant 12$ months would also help to define the usefulness and potential health benefit of pedometers.

\section{Carolyn Raina Elley, MD}

University of Auckland

Auckland, New Zealand

1. Tudor-Locke C, Bassett DR Jr. How many steps/day are enough? Preliminary pedometer indices for public health. Sports Med 2004:34:1-8. 